\title{
Electric-field switching of perpendicularly magnetized multilayers
}

\author{
Yasuhiro Shirahata ${ }^{1}$, Ryota Shiina ${ }^{1}$, Diego López González ${ }^{2}$, Kévin JA Franke ${ }^{2}$, Eiji Wada ${ }^{1}$, Mitsuru Itoh ${ }^{1}$, \\ Nikolay A Pertsev ${ }^{3}$, Sebastiaan van Dijken ${ }^{2}$ and Tomoyasu Taniyama ${ }^{1}$ \\ Perpendicularly magnetized layers are used widely for high-density information storage in magnetic hard disk drives and \\ nonvolatile magnetic random access memories. Writing and erasing of information in these devices is implemented by \\ magnetization switching in local magnetic fields or via intense pulses of electric current. Improvements in energy efficiency \\ could be obtained when the reorientation of perpendicular magnetization is controlled by an electric field. Here, we report on \\ reversible electric-field-driven out-of-plane to in-plane magnetization switching in $\mathrm{Cu} / \mathrm{Ni}$ multilayers on ferroelectric $\mathrm{BaTiO}_{3}$ at \\ room temperature. Fully deterministic magnetic switching in this hybrid material system is based on efficient strain transfer from \\ ferroelastic domains in $\mathrm{BaTiO}_{3}$ and the high sensitivity of perpendicular magnetic anisotropy in $\mathrm{Cu} / \mathrm{Ni}$ to electric-field-induced \\ strain modulations. We also demonstrate that the magnetoelectric coupling effect can be used to realize $180^{\circ}$ magnetization \\ reversal if the out-of-plane symmetry of magnetic anisotropy is temporarily broken by a small magnetic field.
}

NPG Asia Materials (2015) 7, e198; doi:10.1038/am.2015.72; published online 10 July 2015

\section{INTRODUCTION}

Various studies have been published on electric-field-controlled magnetic effects in recent years, including magnetic domain wall propagation, ${ }^{1-8}$ magnetic phase transitions, ${ }^{9-12}$ spin polarization, ${ }^{13,14}$ magnetic anisotropy ${ }^{15-32}$ and exchange bias. ${ }^{33-37}$ Electric-field control of perpendicular magnetic anisotropy (PMA) would open up new prospects for the realization of high-density magnetic memory and logic technologies operating at low energy consumption levels. Attempts to attain this goal have mostly focused on charge accumulation or band shifting in ultrathin ferromagnetic layers with a metal oxide gate dielectric. ${ }^{15-20}$ In these systems, stable reversal of perpendicular magnetization can be realized when precessional motion of magnetization is triggered by short voltage pulses. ${ }^{19,20}$ Giant modulations of PMA have also been obtained by voltage control of oxygen ion migration in metal/metal oxide bilayers. ${ }^{32}$ Another popular approach towards the engineering of electric-field-controlled magnetic properties is based on interfacial mechanical strain coupling between ferromagnetic and ferroelectric materials in multiferroic hybrids. ${ }^{4-6,8,10,12,21-31,38}$ In this case, transfer of lattice strains across interfaces alters the magnetoelastic anisotropy of a ferromagnetic layer via inverse magnetostriction. Anisotropy modulations of more than one order of magnitude have been obtained, which has enabled full electric-field control of the magnetization orientation in two-phase multiferroic systems. However, in spite of intense research efforts in recent years, efficient electric-field control via strain coupling has thus far been limited to ferromagnetic films with in-plane magnetization. Although small modulations of PMA have been shown ${ }^{21,27-29}$ and reversal of local magnetic areas has been demonstrated, ${ }^{30}$ fully deterministic and reversible electric-field-induced switching between perpendicular and in-plane magnetization states in uniform films has remained elusive despite its technological relevance.

Here, we report on purely electric-field-driven $90^{\circ}$ magnetization switching in PMA-Cu/Ni multilayers on $\mathrm{BaTiO}_{3}$ at room temperature. Magnetization rotation in our strain-coupled system is fully reversible and repeatable. Also, we demonstrate that $180^{\circ}$ magnetization reversal is obtained in an electric field when the out-of-plane symmetry of PMA is temporarily broken by a small magnetic field. Electric-fieldinduced magnetic switching is driven by large variations of magnetic anisotropy in the $\mathrm{Cu} / \mathrm{Ni}$ multilayer originating from ferroelastic domain switching in $\mathrm{BaTiO}_{3}$. The electric field that is required to reorient the magnetization is modest and strict limitations on the total multilayer thickness are not imposed. Our results do therefore present a new and promising realization of fully electric-field-controlled magnetization switching in a PMA system.

\section{MATERIALS AND METHODS}

Multilayer growth

$[\mathrm{Cu}(9 \mathrm{~nm}) / \mathrm{Ni}(2 \mathrm{~nm})]_{5} / \mathrm{Cu}(9 \mathrm{~nm})$ multilayers were grown by molecular beam epitaxy on nominally in-plane [100]-poled $\mathrm{BaTiO}_{3}$ single-crystal substrates in an ultrahigh vacuum chamber with a base pressure below $10^{-10}$ Torr. ${ }^{39}$ Before $\mathrm{Cu}$ and $\mathrm{Ni}$ deposition, a 1-nm thin Fe buffer layer was grown at $300^{\circ} \mathrm{C}$. All $\mathrm{Cu}$ and $\mathrm{Ni}$ layers were deposited at room temperature and the multilayers were covered by $5 \mathrm{~nm}$ of Au to prevent oxidation during sample characterization. The growth process was monitored by reflection high-energy electron

${ }^{1}$ Materials and Structures Laboratory, Tokyo Institute of Technology, Yokohama, Japan; ${ }^{2}$ NanoSpin, Department of Applied Physics, Aalto University School of Science, Fl-00076 Aalto, Finland and ${ }^{3}$ A. F. Ioffe Physical-Technical Institute and Peter the Great St. Petersburg Polytechnic University, St. Petersburg, Russia 
a
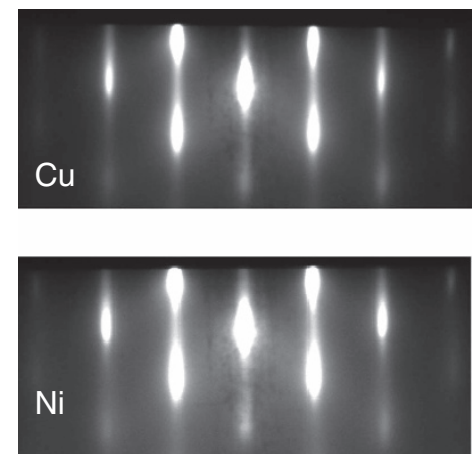

C

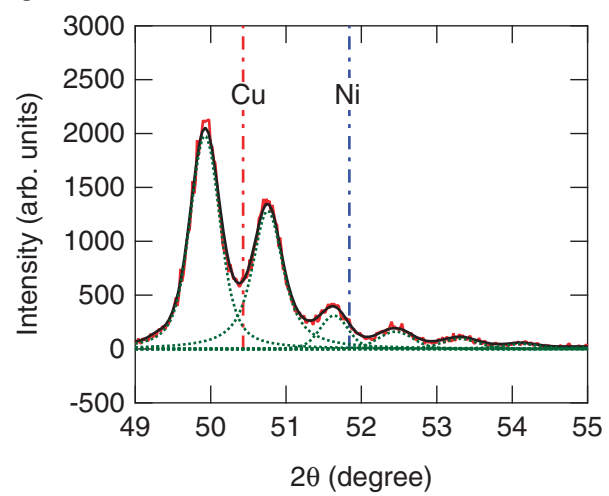

b

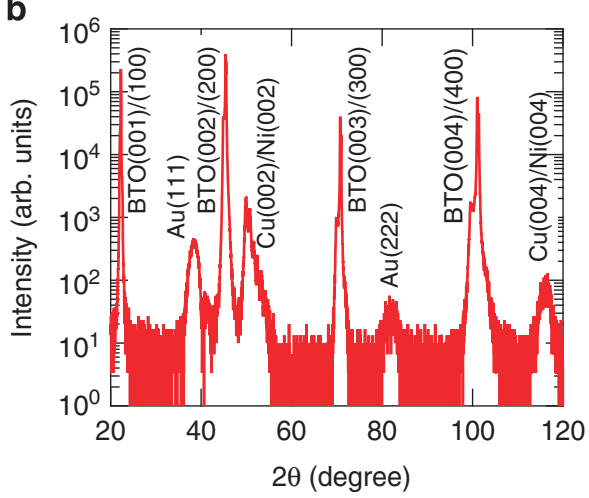

d

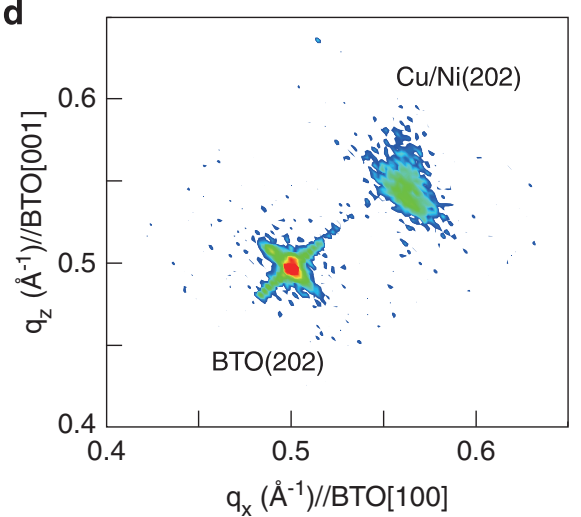

Figure 1 (a) RHEED images of the top $\mathrm{Ni}$ and $\mathrm{Cu}$ layers along the $\langle 100\rangle$ direction of $\mathrm{BaTiO}_{3}$. (b) X-ray diffraction spectrum of a $[\mathrm{Cu}(9 \mathrm{~nm}) / \mathrm{Ni}(2 \mathrm{~nm})]_{5} / \mathrm{Cu}$ $(9 \mathrm{~nm}) / \mathrm{Fe}(1 \mathrm{~nm}) / \mathrm{BaTiO}_{3}(\mathrm{OO})$ heterostructure and (c) an expanded view of an interference fringe from the Cu/Ni multilayer. The black solid curve in $\mathbf{c}$ is a fit to the experimental data using Voigt functions (green dotted lines) to match the satellite peaks. The red and blue dash-dotted lines indicate the diffraction angles of bulk $\mathrm{Cu}(002)$ and $\mathrm{Ni}(002)$. (d) Reciprocal lattice map around the (202) diffraction peaks of the $\mathrm{Cu} / \mathrm{Ni}$ multilayer and $\mathrm{BaTiO}_{3}$ substrate.

diffraction (RHEED) and detailed structural analysis was performed using an $\mathrm{X}$-ray diffraction $(\mathrm{XRD})$ measurement system with $\mathrm{Cu} K \alpha$ radiation.

\section{Magnetic and ferroelectric characterization}

Electric-field-induced magnetic switching in the $\mathrm{Cu} / \mathrm{Ni}$ multilayers was studied using the magneto-optical Kerr effect (MOKE). A laser-based MOKE setup with polar and longitudinal measurement geometries and a laser spot diameter of about $500 \mu \mathrm{m}$ was used to record magnetic hysteresis curves in an out-ofplane electric field of up to $\pm 10 \mathrm{kV} \mathrm{cm}^{-1}$. Time-resolved MOKE measurements were conducted using a digital oscilloscope (WavePro 7100A, Teledyne LeCroy, NY, USA) with a sampling rate of $20 \mathrm{GS} \mathrm{s}^{-1}$ and a bandwidth of $1 \mathrm{GHz}$. MOKE microscopy was performed to visualize the evolution of the magnetic domain structure in an applied electric field and to measure local magnetic hysteresis curves in polar Kerr geometry. Polarization switching in the $\mathrm{BaTiO}_{3}$ substrate was measured using a ferroelectric materials tester (TF Analyzer 2000, aixACCT Systems, Aachen, Germany) and differential interference contrast microscopy.

\section{RESULTS}

\section{Structural characterization}

Figure 1a shows reflection high-energy electron diffraction patterns of the top $\mathrm{Ni}$ and $\mathrm{Cu}$ layers of a $[\mathrm{Cu}(9 \mathrm{~nm}) / \mathrm{Ni}(2 \mathrm{~nm})]_{5} / \mathrm{Cu}(9 \mathrm{~nm}) / \mathrm{Fe}$ $(1 \mathrm{~nm}) / \mathrm{BaTiO}_{3}(001)$ multiferroic heterostructure. The high-energy electron diffraction patterns confirm fully epitaxial film growth with an in-plane orientation of [100] Ni II [100] Cu II [110]Fe II [100] $\mathrm{BaTiO}_{3}$. The X-ray diffraction spectrum of Figure $1 \mathrm{~b}$ confirms that secondary phases are absent in the $\mathrm{Cu} / \mathrm{Ni}$ multilayer. The $\mathrm{BaTiO}_{3}$ substrate exhibits a tetragonal crystal structure at room temperature with lattice constants $a_{\mathrm{BTO}}=3.992 \AA$ and $c_{\mathrm{BTO}}=4.036 \AA$ and a ferroelectric polarization aligned along the $c$ axis. The splitting of the $\mathrm{BaTiO}_{3}$ reflections into $(h 00)$ and $(00 l)$ peaks signifies the coexistence of two types of ferroelectric domains. Domains with in-plane ferroelectric polarization (hereafter referred to as $a$-domains) exhibit a rectangular in-plane lattice structure $\left(x_{1}=a_{\mathrm{BTO}}\right.$ and $\left.x_{2}=c_{\mathrm{BTO}}\right)$ and an out-ofplane lattice spacing of $x_{3}=a_{\mathrm{BTO}}$. The $a$-domains are separated by domains with a perpendicular-to-plane polarization ( $c$-domains). The in-plane lattice structure of the $c$-domains is square $\left(x_{1}=x_{2}=a_{\mathrm{BTO}}\right)$ and $x_{3}=c_{\mathrm{BTO}}$. Since the $\mathrm{BaTiO}_{3}(h 00)$ reflections are more intense than the corresponding $(00 l)$ peaks, the $a$-domains are larger than the $c$-domains in the pristine $\mathrm{BaTiO}_{3}$ substrate. From the integrated intensities of the split peaks, the total sample coverage of $a$-domains is estimated as $93 \%$ in this sample.

Figure 1c shows a clear X-ray diffraction interference fringe from the $\mathrm{Cu} / \mathrm{Ni}$ multilayer. The pronounced satellite peaks are a signature of high structural quality and smooth interfaces. The $\mathrm{Cu} / \mathrm{Ni}$ bilayer thickness $D$ can be calculated from the angular positions of the satellite peaks, which are decomposed into Voigt functions ${ }^{40}$

$$
D=\frac{n \lambda}{2\left|\sin \theta_{n}-\sin \theta_{0}\right|} .
$$

Here, $\theta_{n}$ and $\theta_{0}$ are the diffraction angles of the $n$th order satellite and the fundamental Bragg peak, and $\lambda$ is the X-ray wavelength. Fitting of the experimental data using Equation 1 gives $D=11.6 \mathrm{~nm}$, which is compatible with the nominal layer thickness of the $\mathrm{Cu}(9 \mathrm{~nm}) / \mathrm{Ni}$ $(2 \mathrm{~nm})$ bilayer.

The shift of the $\mathrm{Cu}(002) / \mathrm{Ni}(002)$ reflection from the diffraction angles of bulk $\mathrm{Cu}(002)\left(a_{\mathrm{Cu}}=3.615 \AA\right)$ and bulk Ni(002) $\left(a_{\mathrm{Ni}}=3.524 \AA\right)$ 


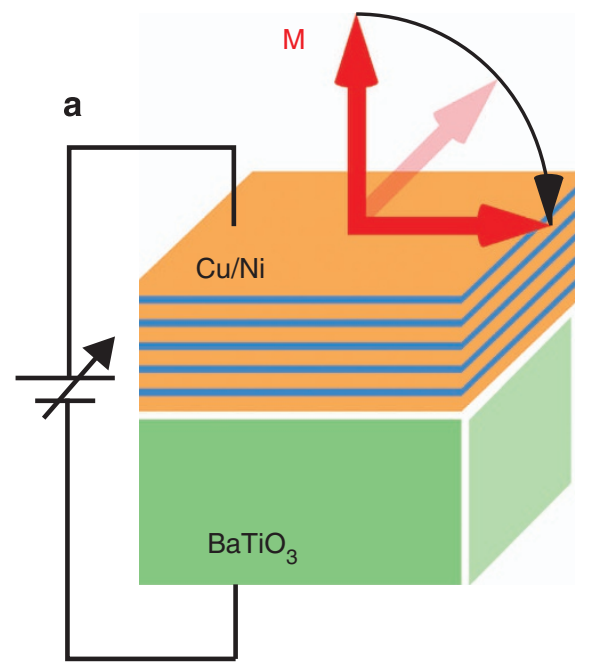

b

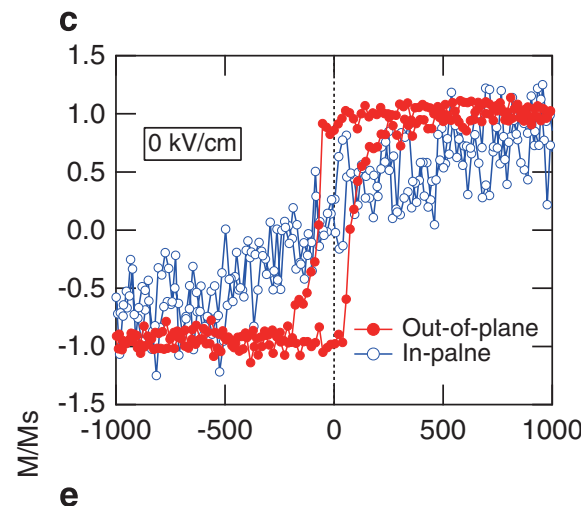

d
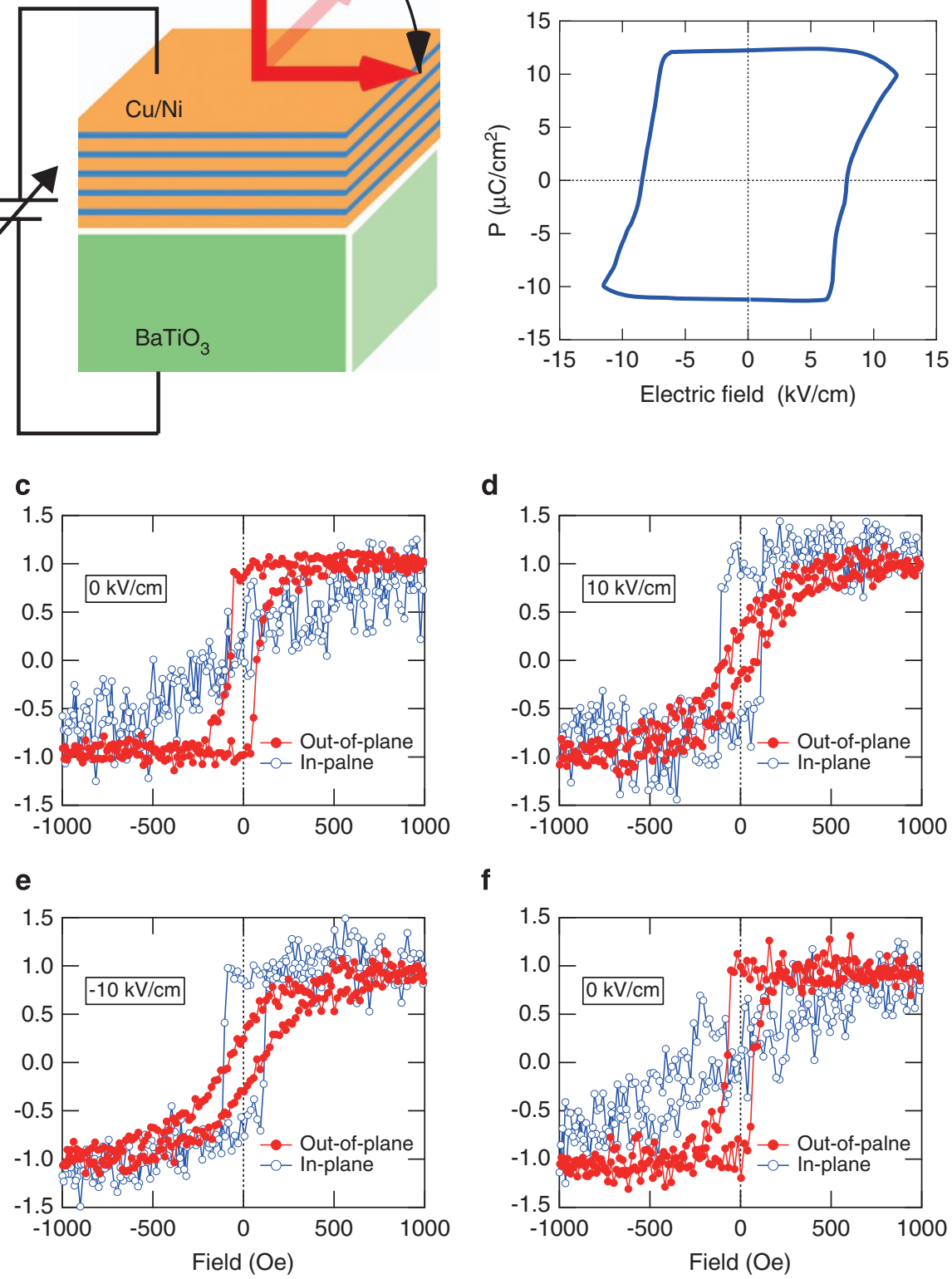

Figure 2 (a) Schematic illustration of the experimental system. (b) Out-of-plane polarization hysteresis curve of the $\mathrm{BaTiO}_{3} \mathrm{substrate}$ (c-f) $\mathrm{Normalized}$ out-of-plane and in-plane magnetic hysteresis curves of the $\mathrm{Cu} / \mathrm{Ni}$ multilayer recorded while sequentially applying an electric field of (c) $E=0 \mathrm{kV} \mathrm{cm}^{-1}$, (d) $E=10 \mathrm{kV} \mathrm{cm}^{-1}$, (e) $E=-10 \mathrm{kV} \mathrm{cm}^{-1}$ and (f) $E=0 \mathrm{kV} \mathrm{cm}^{-1}$ across $\mathrm{BaTiO}_{3}$.

reveals a mean out-of-plane lattice spacing of $3.673 \AA$. Reciprocal lattice maps around the (002) and (202) diffraction peaks indicate a corresponding in-plane lattice spacing of $3.590 \AA$ for the as-grown $\mathrm{Cu} / \mathrm{Ni}$ multilayer on $\mathrm{BaTiO}_{3}$. The lattice strain in the Ni layers is thus tensile and amounts to $1.9 \%$. This experimental value is in excellent agreement with a theoretical calculation based on the actual thicknesses of $\mathrm{Cu}$ $(9 \mathrm{~nm})$ and $\mathrm{Ni}(2 \mathrm{~nm})$ and their elastic stiffness, which yields an in-plane lattice constant of $3.592 \AA$ for a coherent free-standing $\mathrm{Cu} / \mathrm{Ni}$ multilayer. We note that the reciprocal lattice map around the (202) diffraction peak shown in Figure 1d clearly shows that the misfit strain caused by the lattice mismatch between the $\mathrm{Ni} / \mathrm{Cu}$ multilayer and $\mathrm{BaTiO}_{3}$ substrate is relaxed in the epitaxially grown film. A detailed theoretical analysis of strain and magnetic anisotropy is given after the next section.

\section{Electric-field-driven $90^{\circ}$ magnetization switching}

$\mathrm{Cu} / \mathrm{Ni}$ multilayers were selected for this study because they provide strong $\mathrm{PMA}$ in a relatively large $\mathrm{Ni}$ thickness range (typically $\left.1.5 \mathrm{~nm}<t_{f}<8 \mathrm{~nm}\right) .{ }^{41}$ Moreover, since PMA in $\mathrm{Cu} / \mathrm{Ni}$ originates from a magnetoelastic effect, it is predicted to be sensitive to electric-fieldinduced strain modulations. ${ }^{38}$ Figure 2 shows the experimental configuration, polarization switching in $\mathrm{BaTiO}_{3}$, and normalized out-of-plane and in-plane magnetic hysteresis curves for different electric fields across the $\mathrm{BaTiO}_{3}$ substrate. The data in Figures $2 \mathrm{c}-\mathrm{f}$ provide macroscopic information about the magnetic configuration at room temperature and were recorded using a MOKE setup with polar and longitudinal measurement geometries. Before applying any electric field (Figure 2c), a square-shaped out-of-plane hysteresis curve 
is obtained while the in-plane one is slanted and almost completely unopened. These measurements clearly establish that the $\mathrm{Cu} / \mathrm{Ni}$ multilayer exhibits PMA in the as-grown state. The slope of the inplane magnetic hysteresis curve corresponds to a magnetic anisotropy strength of $K_{\text {eff }} \approx 2.4 \times 10^{4} \mathrm{~J} \mathrm{~m}^{-3}$. When a positive electric field of $10 \mathrm{kV} \mathrm{cm}^{-1}$ is applied to $\mathrm{BaTiO}_{3}$ (Figure 2d), the shapes of both hysteresis curves change instantaneously. Now the out-of-plane curve is slanted and the in-plane hysteresis curve is square. This apparent reversal in the shape of the hysteresis curves indicates a rotation of the easy magnetization axis from perpendicular $\left(E=0 \mathrm{kV} \mathrm{cm}^{-1}\right)$ to inplane $\left(E=10 \mathrm{kV} \mathrm{cm}^{-1}\right)$, as illustrated in Figure 2a. A very similar result is obtained for a negative electric field of $-10 \mathrm{kV} \mathrm{cm}^{-1}$ (Figure 2e). The electric-field-induced magnetic switching effect does therefore not depend on the polarity of the applied bias voltage. Finally, when the electric field is switched off (Figure 2f), the magnetization of the $\mathrm{Cu} / \mathrm{Ni}$ multilayer rotates back to a perpendicular direction. We note that the electric-field-controlled switching sequence of Figure 2 is fully repeatable over many on/off cycles of applied bias voltage. Moreover, the magnitude of the electric-field-driven effect is very large. The converse magnetoelectric coupling coefficient $\alpha=\mu_{0} \Delta M_{\mathrm{S}} / E$ amounts to $6 \times 10^{-7} \mathrm{~s} \mathrm{~m}^{-1}$, which is the highest reported magnetoelectric coupling efficiency for a strain-coupled PMA system. Modest electric-field strengths of $\pm 10 \mathrm{kV} \mathrm{cm}^{-1}$ were used in the switching experiments because it suffices to saturate the polarization of the $\mathrm{BaTiO}_{3}$ substrate in a perpendicular-to-plane direction (Figure 2b and Figure 3).

Information about the magnetic microstructure of the $\mathrm{Cu} / \mathrm{Ni}$ multilayer is provided by MOKE microscopy. The images presented in Figure 3a show the evolution of the magnetic domain structure as a function of out-of-plane electric field. In the initial remanent state (1), the sample consists of elongated magnetic stripe domains with alternating perpendicular and mostly in-plane magnetizations. The application of a small electric field of $2 \mathrm{kV} \mathrm{cm}^{-1}$ switches the magnetization into the film plane via fast lateral growth of in-plane magnetized domains (2)-3). The magnetic configuration of (3) does not change upon further increase of the electric-field strength. When the electric field is switched off, perpendicularly magnetized stripe domains are restored (4). Reversal of the out-of-plane electric-field direction first enlarges the perpendicular magnetic domains (5-6)), but the process is suddenly reversed at an electric field of $-2.5 \mathrm{kV}$ $\mathrm{cm}^{-1}$ (7)). A further increase of the electric field promotes the growth of domains with in-plane magnetization (8) until saturation is reached at $E=-4 \mathrm{kV} \mathrm{cm}^{-1}$ (9). Finally, a magnetic configuration with alternating perpendicular and in-plane domains is again reestablished in the $\mathrm{Cu} / \mathrm{Ni}$ multilayer after the electric field is switched off (10). The MOKE microscopy data of Figure 3a indicate that rotation of the easy magnetization axis into the film plane is symmetric in large applied electric fields (3) and (9), in agreement with the measurements of Figure 2. However, for small electric fields (that is, below saturation) the response can be asymmetric, depending on the direction of the applied electric field relative to the ferroelectric polarization in the $\mathrm{BaTiO}_{3}$ substrate. Another observation relates to the orientation of magnetization inside the in-plane domains. Although the unopened and slanted hysteresis curves in (3) and (9) indicate fully in-plane magnetization in saturation, the hysteresis loops of the same domains are not completely closed in the multidomain

a
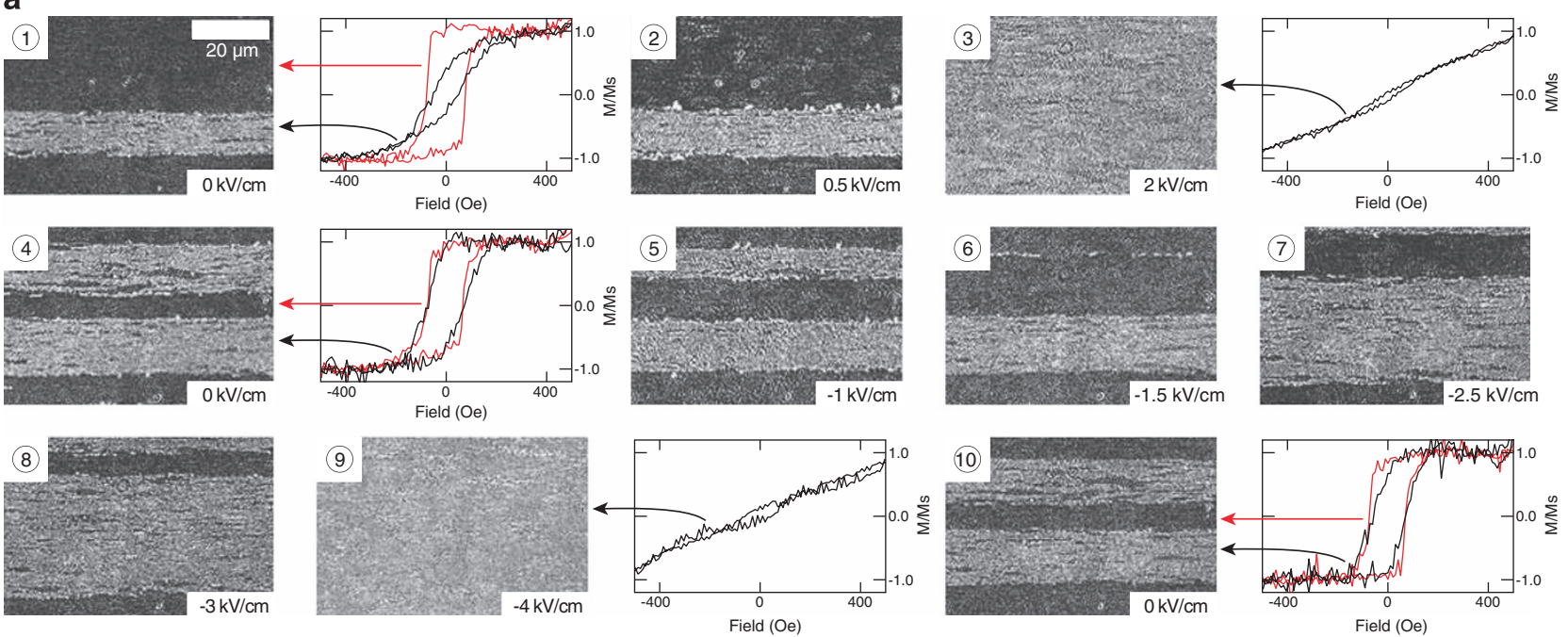

b

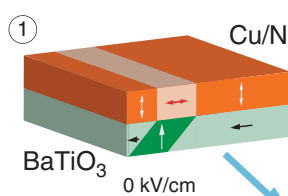

$\mathrm{Cu} / \mathrm{Ni}$

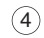

(4)
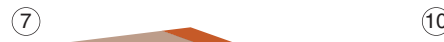

(10)
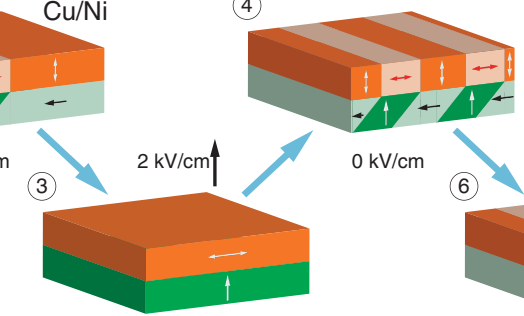

$0 \mathrm{kV} / \mathrm{cm}$
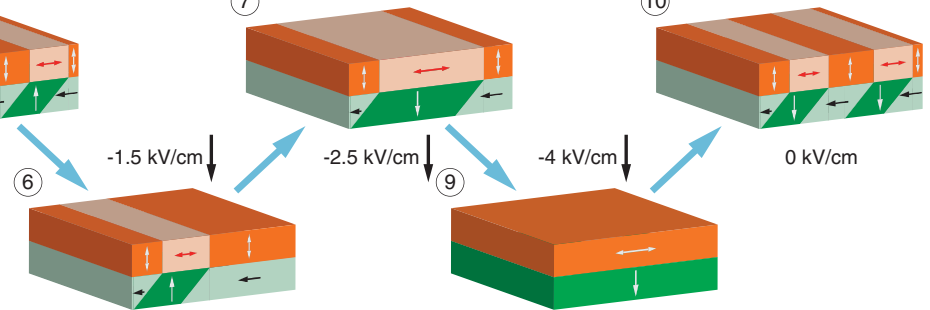

$-2.5 \mathrm{kV} / \mathrm{cm}$

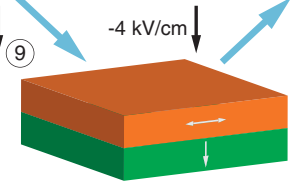

$0 \mathrm{kV} / \mathrm{cm}$

Figure 3 (a) MOKE microscopy images (measured in zero magnetic field) and local polar MOKE curves of the Cu/Ni multilayer as a function of out-of-plane electric field. (b) Schematic illustration of the ferroelectric domain structure in the $\mathrm{BaTiO}_{3}$ substrate (green) and the magnetic domains of the $\mathrm{Cu} / \mathrm{Ni}$ multilayer (orange) during the electric-field-controlled switching sequence in a. 
state. Magnetic contrast within the domains suggest that the magnetization directions are partly perpendicular, which could be caused by coupling to the perpendicular magnetization of neighboring stripe domains.

The electric-field-controlled switching events and magnetic anisotropy modulations are fully explained by considering the ferroelastic domain pattern of the ferroelectric $\mathrm{BaTiO}_{3}$ substrate and interfacial strain transfer to the $\mathrm{Cu} / \mathrm{Ni}$ multilayer. Molecular beam epitaxy of the $\mathrm{Cu} / \mathrm{Ni}$ multilayer onto $\mathrm{BaTiO}_{3}$ produces a fully (001)-oriented layered structure with smooth interfaces. However, owing to the significant differences $(>10 \%)$ between the lattice constants of $\mathrm{BaTiO}_{3}$ and those of $\mathrm{Cu}$ and $\mathrm{Ni}$, most of the lattice mismatch strain relaxes at the onset of $\mathrm{Cu}$ growth. Hereafter, the $\mathrm{Cu} / \mathrm{Ni}$ multilayer grows with an inplane lattice constant of $3.590 \AA$, which corresponds closely to that of a coherent free-standing $\mathrm{Cu} / \mathrm{Ni}$ multilayer. Thus, a $1.9 \%$ tensile strain is induced in the Ni layers, which is determined by the lattice mismatch between $\mathrm{Cu}$ and $\mathrm{Ni}$ and the elastic stiffnesses and thicknesses of $\mathrm{Cu}$ and $\mathrm{Ni}$ layers. From this we conclude that the layers are almost completely decoupled from the ferroelastic $\mathrm{BaTiO}_{3}$ domains in the asgrown state. Since the magnetoelastic coefficient of $\mathrm{Ni}$ is positive, the tensile strain induces PMA in the $\mathrm{Cu} / \mathrm{Ni}$ multilayer.

If an out-of-plane electric field is applied along the direction of ferroelectric polarization in the $c$-domains, these domains grow at the expense of $a$-domains. This ferroelectric switching effect coincides with an abrupt change of the in-plane lattice structure in $\mathrm{BaTiO}_{3}$. In all areas where $a$-domains are replaced by $c$-domains, the lattice constant is reduced by $1.1 \%$ along one of the crystallographic axes. Since the $\mathrm{Cu} / \mathrm{Ni}$ multilayer is firmly clamped to the $\mathrm{BaTiO}_{3}$ substrate, a large fraction of this uniaxial compressive strain is transferred to the $\mathrm{Cu}$ and Ni layers. This electric-field-controlled strain effect opposes the original growth-induced tensile strain state, leading to magnetization reorientation from a perpendicular-to-plane to an in-plane direction. Figure $3 \mathrm{~b}$ illustrates the experimental switching sequence of Figure 3a starting with $c$-domain expansion (1). When the ferroelectric substrate is transformed into a single-domain $c$ state, the easy magnetization direction is oriented in the film plane (3). The $\mathrm{BaTiO}_{3}$ substrate relaxes back to a polydomain state with $a-c$ stripe domains after the electric field is turned off (4)). In this case, reversible strain transfer to the $\mathrm{Cu} / \mathrm{Ni}$ multilayer induces PMA on top of the ferroelectric $a$-domains. When a small electric field is applied against the ferroelectric polarization of the $c$-domains, the $a$-domains grow (6) until the perpendicular polarization in the $c$-domains abruptly aligns with the external electric field by $180^{\circ}$ switching. Afterwards, the $c$-domains grow at the expense of $a$-domains (7) until the singledomain state is reached (9). After the electric field is switched off, relaxation into a structure with $a-c$ domains commences again (10). Throughout this sequence of electric fields, the evolution of the ferroelectric domain structure is mimicked by the domains in the $\mathrm{Cu} / \mathrm{Ni}$ multilayer because of efficient strain transfer and inverse magnetostriction. We note that the electric field that is required to rotate the magnetization in the MOKE microscopy experiments (Figure 3a) is relatively small compared with the switching field in the polarization hysteresis curve of the $\mathrm{BaTiO}_{3}$ substrate (Figure $2 b$ ). This difference could be explained by the time-scale of the two measurements or a sample-to-sample variation. Although the polarization hysteresis curve was obtained at $0.2 \mathrm{~Hz}$, the MOKE microscopy data were typically recorded some $10 \mathrm{~s}$ after the electric field was set. We would also like to point out that the initial state in Figure 3a, (1) was obtained after the application of an electric field and prolonged domain relaxation. Hence, it does not represent the as-grown state. Directly after growth, no domain correlations between the ferroelectric
$\mathrm{BaTiO}_{3}$ substrate and the $\mathrm{Cu} / \mathrm{Ni}$ multilayer exist because of strong relaxation of lattice mismatch strain in the first $\mathrm{Cu}$ layer. As a result, as-grown samples exhibit uniform PMA irrespective of the underlying ferroelectric domain pattern.

\section{Theoretical analysis of strain and magnetic anisotropy}

To confirm that interfacial strain transfer from ferroelastic domains in $\mathrm{BaTiO}_{3}$ to the $\mathrm{Cu} / \mathrm{Ni}$ multilayer explains the experimental results, we analyze the magnetization-dependent part of the free energy of a strained Ni layer written as a function of the direction cosines $m_{i}$ $(i=1,2,3)$ of the unit vector $\mathbf{m}=\mathbf{M} / M_{s}$. In our formulation, the interfacial magnetic anisotropy is incorporated into the volumetric energy density $\Delta F\left(m_{i}\right)$ via the term $K_{s} m_{3}^{2} / t_{f}$, where $t_{f}$ is the Ni layer thickness and $K_{\mathrm{s}}$ is the parameter characterizing the sum of specific energies of two $\mathrm{Ni} / \mathrm{Cu}$ interfaces. In the crystallographic reference frame with the $x_{3}$ axis orthogonal to the interfaces, the resulting energy density $\Delta F\left(m_{i}\right)$ can be written as ${ }^{42}$

$$
\begin{aligned}
& \Delta F \cong K_{1 \|} m_{1}^{2} m_{2}^{2}+K_{1 \perp}\left(m_{1}^{2}+m_{2}^{2}\right) m_{3}^{2}+\frac{K_{s}}{t_{f}} m_{3}^{2}+B_{1}\left(u_{1} m_{1}^{2}+u_{2} m_{2}^{2}\right) \\
& -B_{1}\left[\frac{B_{1}}{6 c_{11}}+\frac{c_{12}}{c_{11}}\left(u_{1}+u_{2}\right)\right] m_{3}^{2}+\frac{1}{2} \mu_{0} M_{s}^{2}\left(N_{11} m_{1}^{2}+N_{22} m_{2}^{2}+N_{33} m_{3}^{2}\right),
\end{aligned}
$$

where $K_{1 \|}\left(t_{f}\right)$ and $K_{1 \perp}\left(t_{f}\right)$ are the in-plane and out-of-plane magnetocrystalline anisotropy constants of fourth order, ${ }^{43} \mu_{0}$ is the permeability of vacuum, $N_{i i}$ are the diagonal components of the tensor of demagnetizing factors, $u_{1}$ and $u_{2}$ are the in-plane normal strains (shear strains are absent in our case), $B_{1}$ is the magnetoelastic coefficient ${ }^{44}$ and $c_{11}$ and $c_{12}$ are the elastic stiffnesses at fixed $\mathbf{M}$ (we use the Voigt matrix notation for strains and elastic constants). Using the relations $m_{1}=\cos \phi \sin \theta, m_{2}=\sin \phi \sin \theta$ and $m_{3}=\cos \theta$, we express the energy density $\Delta F$ through the polar angle $\theta$ and the azimuth angle $\phi$ of the magnetization direction. Numerical minimization of the function $\Delta F$ $(\phi, \theta)$ allows us to determine the equilibrium magnetization orientations in the cubic Ni layers for different in-plane lattice strains $u_{1}$ and $u_{2}$. We performed this minimization for Ni films with $t_{f}=2 \mathrm{~nm}$ and large in-plane dimensions ensuring $N_{11}=N_{22}=0$ and $N_{33}=1$ using the following values of the involved material parameters: $M_{\mathrm{s}}=4.85 \times 10^{5} \mathrm{~A} \mathrm{~m}^{-1},{ }^{44} K_{\mathrm{s}}=4.3 \times 10^{-4} \mathrm{~J} \mathrm{~m}^{-2},{ }^{45} K_{1 \|}=-2.510^{4} \mathrm{~J} \mathrm{~m}^{-3}$, $K_{1 \perp}=-1.2 \times 10^{4} \mathrm{~J} \mathrm{~m}^{-3},{ }^{43} B_{1}=9.2 \times 10^{6} \mathrm{~J} \mathrm{~m}^{-3}, 46 c_{11}=2.465 \times 10^{11} \mathrm{~J} \mathrm{~m}^{-2}$ and $c_{12}=1.473 \times 10^{11} \mathrm{~J} \mathrm{~m}^{-2} .47$ In the free-standing multilayer, the lattice matching between the 2-nm thick Ni layers and the 9-nm thick $\mathrm{Cu}$ films produces tensile strains $u_{1}=u_{2} \cong 1.9 \%$ in Ni. (This theoretical value is smaller than the nominal misfit strain of $2.58 \%$ because $\mathrm{Cu}$ layers are slightly compressively strained by $\mathrm{Ni}$ ones in elastic equilibrium.) Since the magnetoelastic coefficient $B_{1}$ is positive, these strains stabilize a perpendicular magnetization orientation in the $\mathrm{Ni}$ layers $\left(\theta=0\right.$ or $\left.180^{\circ}\right)$. The mechanical interaction between the $\mathrm{Ni} / \mathrm{Cu}$ multilayer and a thick $\mathrm{BaTiO}_{3}$ substrate creates additional strains $\delta u_{1}$ and $\delta u_{2}$ in the Ni layers. Owing to significant differences between the lattice constants of $\mathrm{BaTiO}_{3}$ and those of $\mathrm{Cu}$, the nominal values $\delta u_{i}^{0}$ of these tensile strains appear to be very big $\left(\delta u_{1}^{0} \approx 12 \%\right.$ and $\left.\delta u_{2}^{0} \approx 11 \%\right)$. The actual substrate-induced strains, however, are much smaller because of effective strain relaxation during the $\mathrm{Cu} / \mathrm{Ni}$ multilayer growth. This effect may be described by the relations $\delta u_{1}=\eta \delta u_{1}^{0}$ and $\delta u_{2}=\eta \delta u_{2}^{0}$ with $\eta \ll 1$. Since the strains $\delta u_{1}$ and $\delta u_{2}$ are tensile, the magnetization of the $\mathrm{Ni} / \mathrm{Cu}$ multilayer grown on $\mathrm{BaTiO}_{3}$ initially should have a perpendicular orientation irrespective of the parameter $\eta$. For $\eta=0$, the model gives $a_{\mathrm{Ni}} \cong 3.592 \AA$ and $K_{\text {eff }} \approx 2.5 \times 10^{4} \mathrm{~J} \mathrm{~m}^{-3}$, which are in excellent agreement with the experimental data $\left(a_{\mathrm{Ni}}=3.590 \AA\right.$ and $\left.K_{\text {eff }}=2.4 \times 10^{4} \mathrm{~J} \mathrm{~m}^{-3}\right)$. 
After electric-field-induced switching from an $a$-domain to a $c$-domain, the strain $\delta u_{1}$ becomes $\delta u_{1}=\eta \delta u_{1}^{0}+\xi\left(\delta u_{2}^{0}-\delta u_{1}^{0}\right)$. Here, the strain transfer parameter $\xi$ is expected to be much larger than $\eta$ because of strong interfacial mechanical coupling between the $\mathrm{Cu} / \mathrm{Ni}$

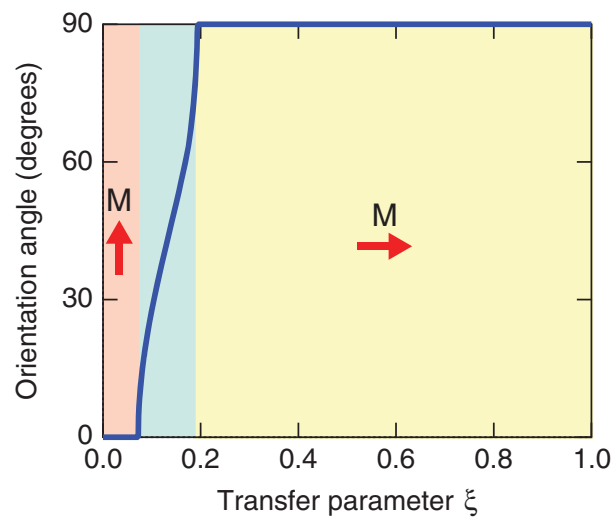

Figure 4 Calculated orientation of the equilibrium magnetization direction in the $\mathrm{Cu} / \mathrm{Ni}$ multilayer after $a$ - to $\mathrm{c}$-domain switching in $\mathrm{BaTiO}_{3}$. The parameter $\xi$ indicates the efficiency of strain transfer from the $\mathrm{BaTiO}_{3}$ substrate to 2-nm thick Ni layers during polarization switching. multilayer and the $\mathrm{BaTiO}_{3}$ substrate during ferroelectric domain switching. Taking $\eta=0$ in first approximation, we calculated the equilibrium magnetization orientation after $90^{\circ}$ polarization switching in $\mathrm{BaTiO}_{3}$ as a function of the transfer parameter $\xi$. Figure 4 shows that, at $\xi<0.073$, the magnetization retains its initial perpendicular direction, whereas at $\xi>0.193$ it acquires an in-plane orientation $\left(\theta=90^{\circ}\right)$. In the intermediate range of $0.073 \leqslant \xi \leqslant 0.193$ the Ni layers should have a canted magnetization with the polar angle $\theta$ gradually increasing with $\xi$ from $0^{\circ}$ to $90^{\circ}$. However, due to efficient transfer of piezoelectric strains, $\xi$ is expected to be close to unity in our experimental system. ${ }^{4}$ For this scenario the model predicts the formation of two types of magnetic areas in an out-of-plane electric field; one with in-plane magnetization (where the ferroelectric domains underneath have switched from $a$ - to $c$-type) and another with perpendicular magnetization (where the ferroelectric domains underneath remain the same as during $\mathrm{Cu} / \mathrm{Ni}$ multilayer growth). This qualitatively agrees with the MOKE microscopy observations in Figure 3. The theoretical model thus fully supports the experimental observations. We note that other magnetoelectric coupling effects, such as electrostatic charge modulation near the $\mathrm{BaTiO}_{3}$ interface, can be excluded as an explanation for electric-field-controlled switching in the $\mathrm{Cu} / \mathrm{Ni}$ multilayer. First, the symmetric magnetic response in a

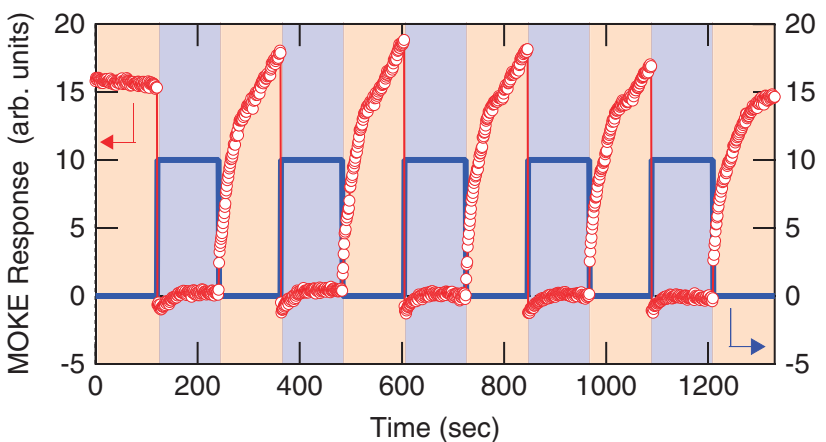

b

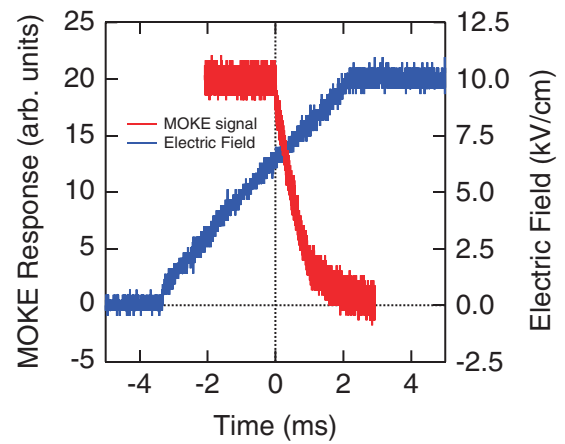

Figure 5 (a) Time-resolved polar MOKE response of the $\mathrm{Cu} / \mathrm{Ni}$ multilayer on $\mathrm{BaTiO}_{3}$ during on and off switching of a $10 \mathrm{kV} \mathrm{cm}^{-1}$ electric field. (b) Zoom-in of the MOKE response during the application of a positive electric-field pulse.

a

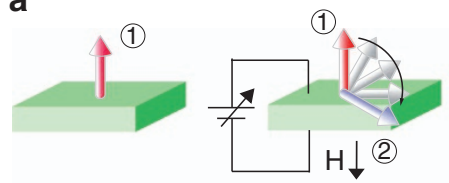

b

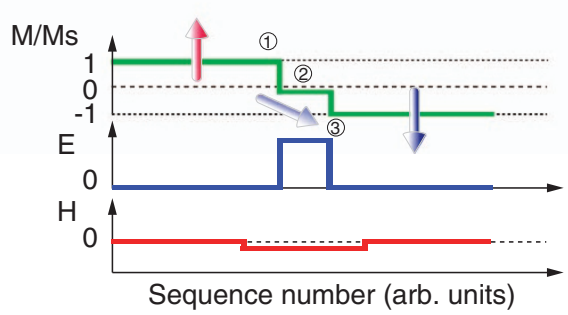

C
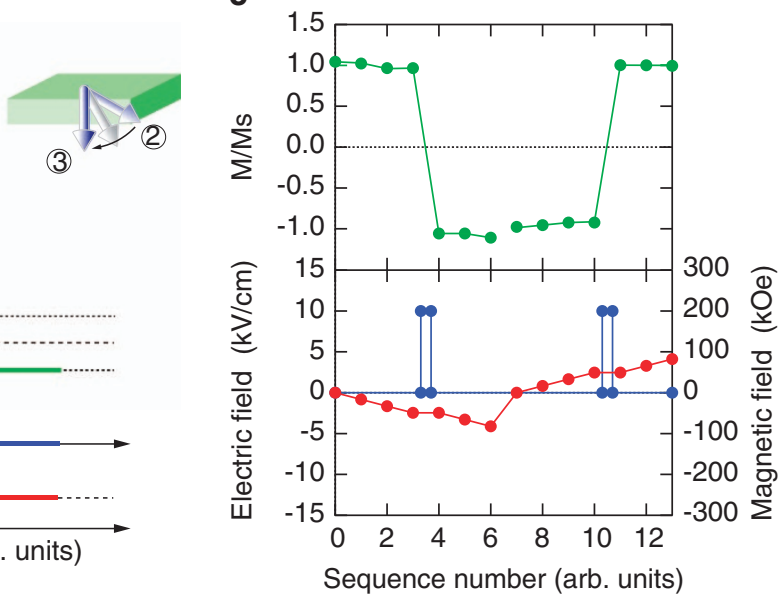

Figure 6 (a) Schematic illustrations of the $180^{\circ}$ magnetization reversal process. (b) Deterministic reversal is obtained by short electric field pulses when a small out-of-plane magnetic field opposes the initial magnetization direction. (c) Experimental demonstration of $180^{\circ}$ magnetization reversal with an electric field of $10 \mathrm{kV} \mathrm{cm}^{-1}$ and a magnetic field of \pm 50 Oe. 
positive and negative electric fields opposes the polarity dependence of charge accumulation. Second, the first $\mathrm{Ni}$ layer and the $\mathrm{BaTiO}_{3}$ substrate are separated by a $9-\mathrm{nm}$ thick $\mathrm{Cu}$ spacer layer, which exceeds the Thomas-Fermi screening length by more than one order of magnitude.

\section{Time-resolved measurements}

The dynamics of electric-field-induced magnetic switching was studied by recording the time-resolved MOKE response of strain-coupled $\mathrm{Cu} / \mathrm{Ni}$ multilayers during the application of a series of voltage pulses across the $\mathrm{BaTiO}_{3}$ substrate. Figure 5 a shows the result for five on/off cycles with an electric field of $10 \mathrm{kV} \mathrm{cm}^{-1}$. Initially, the sample consists of alternating domains with perpendicular and partly in-plane magnetizations (similar to (4) and (10) in Figure 3a). In this state, the polar MOKE signal is large. When an out-of-plane electric field is applied, the MOKE response rapidly drops to zero, indicating fast and complete reorientation of the magnetization into the film plane. After the electric field is turned off, the initial magnetization state is reestablished. Back and forth switching between the two magnetization states is thus reversible and repeatable. The results of Figure $5 \mathrm{a}$ are again explained by ferroelastic domain transformations in $\mathrm{BaTiO}_{3}$. Importantly, they indicate that the time-scale of strain-driven magnetization reorientation is determined by the rate of electric-field induced $a \rightarrow c$ domain switching in $\mathrm{BaTiO}_{3}$, but not by magnetization dynamics itself. Indeed, as can be seen from Figure 5b, the change in polar MOKE signal commences when the electric field reaches $\sim 7 \mathrm{kV} \mathrm{cm}^{-1}$, which closely corresponds to the onset of polarization reversal in $\mathrm{BaTiO}_{3}$ (Figure 2b). Beyond this, the magnetization rapidly reorients into the film plane within $\sim 2 \mathrm{~ms}$. Since the rise time of the electric-field pulse is $\sim 5 \mathrm{~ms}$, the magnetization switching time in this experiment might be limited by the shape of the voltage pulse. Therefore, it should be considered as an upper bound for electricfield-induced magnetic switching in $\mathrm{Cu} / \mathrm{Ni}$ multilayers. Magnetization reorientation in the off-state (Figure $5 \mathrm{a}$ ) is not driven by an electric field but rather by ferroelastic domain relaxation in the $\mathrm{BaTiO}_{3}$ substrate, which proceeds more slowly. The small differences in polar MOKE signal after $120 \mathrm{~s}$ in the off-state are attributed to minor differences in the ferroelectric $a-c$ domain patterns during subsequent relaxation processes.

\section{Electric-field-controlled $180^{\circ}$ magnetization reversal}

Full $180^{\circ}$ reversal between two perpendicular magnetization states can be induced by an electric field if the symmetry of PMA in the $\mathrm{Cu} / \mathrm{Ni}$ multilayers is broken by a small out-of-plane magnetic field. As previously discussed, the application of an electric field to the $\mathrm{BaTiO}_{3}$ substrate reorients the magnetization into the film plane $\left(90^{\circ}\right.$ switching effect). Without an external magnetic field, the magnetization switches back to its original perpendicular position after the electric field is turned off (see Figure 5). However, if a small magnetic field is applied in the opposite out-of-plane direction during the electric-fieldcontrolled magnetic switching sequence, full magnetization reversal by $180^{\circ}$ is realized. In this instance, the magnetization first rotates by $>90^{\circ}$ to a slightly tilted position when the electric field is turned on, and it continues to rotate in the same direction after the electric field is turned off. Figures $6 \mathrm{a}$ and b schematically illustrate the $180^{\circ}$ switching sequence. The experimental MOKE data of Figure $6 \mathrm{c}$ demonstrate that full magnetization reversal in $\mathrm{Cu} / \mathrm{Ni}$ multilayers on $\mathrm{BaTiO}_{3}$ is indeed obtained. Initially, the magnetization of the multilayer points into one of the perpendicular-to-plane directions $\left(M / M_{S}=1\right)$. The application of a small negative magnetic field of $-50 \mathrm{Oe}$ and an electric-field pulse of $10 \mathrm{kV} \mathrm{cm}^{-1}$ fully reverses the magnetization $\left(M / M_{S}=-1\right)$. In Figure $6 c$, we should note that a decrease in the magnetic field down to -50 Oe does not change the magnetization orientation $\left(M / M_{S}=1\right)$, whereas the electric-field pulse suddenly reverses the magnetization to $M / M_{\mathrm{S}}=-1$ at the constant negative magnetic field of $-50 \mathrm{Oe}$. After the electric-field-induced magnetization reversal, a further decrease in magnetic field beyond the magnetic coercive field of $\sim 100$ Oe does not change the magnetization values any more. The results clearly corroborate that the magnetization is fully reversed purely by the electric-field pulse. Back-switching is realized when an opposite magnetic field and the same electric-field pulse are applied $\left(M / M_{S}=1\right)$. The $180^{\circ}$ magnetic switching effect is thus reversible and it only occurs when a small magnetic field is applied against the original perpendicular-to-plane magnetization direction. Similar to $90^{\circ}$ magnetization reorientation, the polarity of the electric field does not affect the $180^{\circ}$ switching effect.

\section{CONCLUSIONS}

In summary, we have demonstrated reversible electric-field control of magnetic switching in epitaxial $\mathrm{Cu} / \mathrm{Ni}$ multilayers with PMA on $\mathrm{BaTiO}_{3}$ substrates. Magnetization reversal in this multiferroic heterostructure is driven by efficient interfacial mechanical strain coupling between the $\mathrm{Cu}$ and $\mathrm{Ni}$ layers and the ferroelastic domains of $\mathrm{BaTiO}_{3}$. Only modest electric fields are required to reorient the magnetization. In addition, the coupling mechanism provides full electric-field control of relatively thick magnetic films. The total multilayer thickness in the reported experiments amounts to $65 \mathrm{~nm}$. Electric-field manipulation of multilayers with similar thicknesses would not be possible by other magnetoelectric coupling effects. The demonstrated ability to deterministically switch relatively thick magnetic films with PMA in small electric fields is potentially interesting for magnetic memories and other devices that could functionally utilize electric-field-controlled manipulation of PMA (for example, in microwave electronics or magnonics). Another property of $\mathrm{Cu} / \mathrm{Ni}$ multilayers, namely giant magnetoresistance, ${ }^{48}$ might also prove useful in this respect, because it renders possible nondestructive readout of local magnetization states.

\section{CONFLICT OF INTEREST}

The authors declare no conflict of interest.

\section{ACKNOWLEDGEMENTS}

This work was supported in part by Industrial Technology Research Grant Program in 2009 from NEDO, Toray Science Foundation, JSPS KAKENHI (Grant Nos. 24.7390, 25.03065), the Advanced Materials Development and Integration of Novel Structured Metallic and Inorganic Materials Project of MEXT, the European Research Council (ERC-2012-StG 307502-E-CONTROL) and the Collaborative Research Project of the Materials and Structures Laboratory, Tokyo Institute of Technology. YS thanks JSPS Fellowships for Young Scientists and KJAF acknowledges financial support from the Finnish Doctoral Program in Computational Sciences. The work at the Ioffe Physical-Technical Institute was supported by the Government of the Russian Federation through the Program P220 (Project No.14.B25.31.0025).

1 Bauer, U., Emori, S. \& Beach, G. S. D. Electric field control of domain wall propagation in Pt/Co/GdOx films. Appl. Phys. Lett. 100, 192408 (2012).

2 Schellekens, A. J., van den Brink, A., Franken, J. H., Swagten, H. J. M. \& Koopmans, B. Electric-field control of domain wall motion in perpendicularly magnetized materials. Nat. Commun. 3, 847 (2012).

3 Chiba, D., Kawaguchi, M., Fukami, S., Ishiwata, N., Shimamura, K. \& Ono, T. Electricfield control of magnetic domain-wall velocity in ultrathin cobalt with perpendicular magnetization. Nat. Commun. 3, 888 (2012).

4 Lahtinen, T. H. E., Franke, K. J. A. \& van Dijken, S. Electric-field control of magnetic domain wall motion and local magnetization reversal. Sci. Rep. 2, 258 (2012). 
5 De Ranieri, E., Roy, P. E., Fang, D., Vehsthedt, E. K., Irvine, A. C., Heiss, D., Casiraghi, A., Campion, R. P., Gallagher, B. L., Jungwirth, T. \& Wunderlich, J. Piezoelectric control of the mobility of a domain wall driven by adiabatic and non-adiabatic torques. Nat. Mater. 12, 808-814 (2012).

6 Lei, N., Devolder, T., Agnus, G., Aubert, P., Daniel, L., Kim, J.-V., Weisheng, Z., Trypiniotis, T., Cowburn, R. P., Chappert, C., Ravelosona, D. \& Lecoeur, P. Straincontrolled magnetic domain wall propagation in hybrid piezoelectric/ferromagnetic structures. Nat. Commun. 4, 1378 (2013).

7 Bauer, U., Emori, S. \& Beach, G. S. D. Voltage-controlled domain wall traps in ferromagnetic nanowires. Nat. Nanotechnol. 8, 441-416 (2013).

8 Franke, K. J. A., Van de Wiele, B., Shirahata, Y., Hamalainen, S. J., Taniyama, T. \& van Dijken, S. Reversible electric-field-driven magnetic domain wall motion. Phys. Rev. X 5, 011010 (2015).

9 Ohno, H., Chiba, D., Matsukura, F., Omiya, T., Abe, E., Dietl, T., Ohno, Y. \& Ohtani, K. Electric-field control of ferromagnetism. Nature 408, 944-946 (2000).

10 Thiele, C., Dörr, K., Bilani, O., Rödel, J. \& Schultz, L. Influence of strain on the magnetization and magnetoelectric effect in $\mathrm{La}_{0.7} \mathrm{~A}_{0.3} \mathrm{MnO}_{3} / \mathrm{PMN}-\mathrm{PT}(001)(\mathrm{A}=\mathrm{Sr}, \mathrm{Ca})$. Phys. Rev. B 75, 054408 (2007)

11 Vaz, C. A. F., Segal, Y., Hoffman, J., Grober, R. D., Walker, F. J. \& Ahn, C. H. Temperature dependence of the magnetoelectric effect in $\mathrm{Pb}\left(\mathrm{Zr}_{0 .}{ }_{2} \mathrm{Ti}_{0.8}\right) \mathrm{O}_{3} /$ $\mathrm{La}_{0.8} \mathrm{Sr}_{0.2} \mathrm{MnO}_{3}$ multiferroic heterostructures. Appl. Phys. Lett. 97, 042506 (2010).

12 Cherifi, R. O., Ivanovskaya, V., Phillips, L. C., Zobelli, A., Infante, I. C., Jacquet, E., Garcia, V., Fusil, S., Briddon, P. R., Guiblin, N., Mougin, A., Ünal, A. A., Kronast, F., Valencia, S., Dkhil, B., Barthélémy, A. \& Bibes, M. Electric-field control of magnetic order above room temperature. Nat. Mater. 13, 345-351 (2014).

13 Garcia, V., Bibes, M., Bocher, L., Valencia, S., Kronast, F., Crassous, A., Moya, X., Enouz-Vedrenne, S., Gloter, A., Imhoff, D., Deranlot, C., Mathur, N. D., Fusil, S. Bouzehouane, K. \& Barthélémy, A. Ferroelectric control of spin polarization. Science 327, 1106-1110 (2010).

14 Pantel, D., Goetze, S., Hesse, D. \& Alexe, M. Reversible electrical switching of spin polarization in multiferroic tunnel junctions. Nat. Mater. 11, 289-293 (2012).

15 Maruyama, T., Shiota, Y., Nozaki, T., Ohta, K., Toda, N., Mizuguchi, M., Tulapurkar, A. A., Shinjo, T., Shiraishi, M., Mizukami, S., Ando, Y. \& Suzuki, Y. Large voltage-induced magnetic anisotropy change in a few atomic layers of iron. Nat. Nanotechnol. 4, 158-161 (2009).

16 Shiota, Y., Maruyama, T., Nozaki, T., Shinjo, T., Shiraishi, M. \& Suzuki, Y. Voltage-assisted magnetization switching in ultrathin $\mathrm{Fe}_{80} \mathrm{CO}_{20}$ alloy layers. Appl. Phys. Express 2, 063001 (2009).

17 Yamada, K., Kakizakai, H., Shimamura, K., Kawaguchi, M., Fukami, S., Ishiwata, N. Chiba, D. \& Ono, T. Electric field modulation of magnetic anisotropy in $\mathrm{MgO} / \mathrm{Co} / \mathrm{Pt}$ structure. Appl. Phys. Express 6, 073004 (2013).

18 Kikuchi, Y., Seki, T., Kohda, M., Nitta, J. \& Takanashi, K. Voltage-induced coercivity change in FePt/MgO stacks with different FePt thicknesses. J. Phys. D: Appl. Phys. 46, 285002 (2013)

19 Kanai, S., Yamanouchi, M., Ikeda, S., Nakatani, Y., Matsukura, F. \& Ohno, H. Electric field-induced magnetization reversal in a perpendicular-anisotropy CoFeB-MgO magnetic tunnel junction. Appl. Phys. Lett. 101, 122403 (2012).

20 Shiota, Y., Nozaki, T., Bonell, F., Murakami, S., Shinjo, T. \& Suzuki, Y. Induction of coherent magnetisation switching in a few atomic layers of FeCo using voltage pulses. Nat. Mater. 11, 39-43 (2012).

21 Lee, J.-W., Shin, S.-C. \& Kim, S.-K. Spin engineering of CoPd alloy films via the inverse piezoelectric effect. Appl. Phys. Lett. 82, 2458-2460 (2003).

22 Eerenstein, W., Wiora, M., Prieto, J. L., Scott, J. F. \& Mathur, N. D. Giant sharp and persistent converse magnetoelectric effects in multiferroic epitaxial heterostructures. Nat. Mater. 6, 348-358 (2007).

23 Sahoo, S., Polisetty, S., Duan, C.-G., Jaswal, S. S., Tsymbal, E. Y. \& Binek, C. Ferroelectric control of magnetism in $\mathrm{BaTiO}_{3} / \mathrm{Fe}$ heterostructures via interface strain coupling. Phys. Rev. B 76, 092108 (2007).

24 Lahtinen, T. H. E., Tuomi, J. O. \& van Dijken, S. Pattern transfer and electric-field induced magnetic domain formation in multiferroic heterostructures. Adv. Mater. 23 3187-3191 (2011).

25 Venkataiah, G., Shirahata, Y., Itoh, M. \& Taniyama, T. Manipulation of magnetic coercivity of $\mathrm{Fe}$ film in $\mathrm{Fe} / \mathrm{BaTiO}_{3}$ heterostructure by electric field. Appl. Phys. Lett. 99 102506 (2011)

26 Brivio, S., Petti, D., Bertacco, R. \& Cezar, J. C. Electric field control of magnetic anisotropies and magnetic coercivity in $\mathrm{Fe} / \mathrm{BaTiO}_{3}(\mathrm{OO})$ heterostructures. Appl. Phys. Lett. 98, 092505 (2011).
27 Lei, N., Park, S., Lecoeur, P., Ravelosona, D., Chappert, C., Stelmakhovych, O. \& Holy, V. Magnetization reversal assisted by the inverse piezoelectric effect in Co-Fe-B/ ferroelectric multilayers. Phys. Rev. B 84, 012404 (2011)

28 Hsu, C. -J., Hockel, J. L. \& Carman, G. P. Magnetoelectric manipulation of domain wall configuration in thin film Ni/[Pb( $\left.\left.\mathrm{Mn}_{1 /} \mathrm{Nb}_{2 \beta}\right) 03\right] 0.68-\left[\mathrm{PbTiO}_{3}\right] 0.32(001)$ heterostructure. Appl. Phys. Lett. 100, 092902 (2012).

29 Tsai, W.-C., Liao, S.-C., Huang, K.-F., Wang, D.-S. \& Lai, C.-H. Nonvolatile electric-field modulation of magnetic anisotropy in perpendicularly magnetized $\mathrm{L}_{0}-\mathrm{FePt} /(001)[\mathrm{Pb}$ $\left.\left(\mathrm{Mg}_{1 \beta} \mathrm{Nb}_{2 \beta}\right)\right]_{0.7}-\left(\mathrm{PbTiO}_{3}\right)_{0.3}$ heterostructures. Appl. Phys. Lett. 103, 252405 (2013)

30 Ghidini, M., Pellicelli, R., Prieto, J. L., Moya, X Soussi, J., Briscoe, J., Dunn, S. \& Mathur, N. D. Non-volatile electrically driven repeatable magnetisation reversal with no applied magnetic field. Nat. Commun. 4, 1453 (2012).

31 Geprägs, S., Mannix, D., Opel, M., Goennenwein, S. T. B. \& Gross, R. Converse magnetoelectric effects in $\mathrm{Fe}_{3} \mathrm{O}_{4} / \mathrm{BaTiO}_{3}$ multiferroic hybrids. Phys. Rev. B $8 \mathbf{8}$ 054412 (2013)

32 Bauer, U., Yao, L., Tan, A. J., Agrawal, P., Emori, S., Tuller, H. L., van Dijken, S. \& Beach, G. S. D. Magneto-ionic control of interfacial magnetism. Nat. Mater. 14, 174-181 (2015)

33 Borisov, P., Hochstrat, A., Chen, X., Kleemann, W. \& Binek, C. Magnetoelectric switching of exchange bias. Phys. Rev. Lett. 94, 117203 (2005).

$34 \mathrm{He}$, X., Wang, Y., Wu, N., Caruso, A. N., Vescovo, E., Belashchenko, K. D. Dowben, P. A. \& Binek, C. Robust isothermal electric control of exchange bias at room temperature. Nat. Mater. 9, 579-585 (2010).

35 Wu, S. M., Cybart, S. A., Yu, P., Rossell, M. D., Zhang, J. X., Ramesh, R. \& Dynes, R. C. Reversible electric control of exchange bias in a multiferroic field-effect device. Nat. Mater. 9, 756-761 (2010).

36 Liu, M., Lou, J., Li, S. \& Sun, N. X. E-field control of exchange bias and deterministic magnetization switching in AFM/FM/FE multiferroic heterostructures. Adv. Func. Mater. 21, 2593-2598 (2011).

37 Wu, S. M., Cybert, S. A., Yi, D., Parker, J. M., Ramesh, R. \& Dynes, R. C. Full electric control of exchange bias. Phys. Rev. Lett. 110, 067202 (2013).

38 Pertsev, N. A. Giant magnetoelectric effect via strain-induced spin reorientation transitions in ferromagnetic films. Phys. Rev. B 78, 212102 (2008).

39 Shirahata, Y., Wada, E., Itoh, M. \& Taniyama, T. Perpendicularly magnetized spin filtering Cu/Ni multilayers. Appl. Phys. Lett. 104, 032404 (2014).

40 Ruotolo, A., Bell, C., Leung, C. W. \& Blamire, M. G. Perpendicular magnetic anisotropy and structural properties of $\mathrm{NiCu} / \mathrm{Cu}$ multilayers. J. Appl. Phys. 96, 512-518 (2004).

41 Johnson, M. T., Bloemen, P. J. H., den Broeder, F. J. A. \& de Vries, J. J. Magnetic anisotropy in metallic multilayers. Rep. Prog. Phys 59, 1409-1458 (1996).

42 Pertsev, N. A. \& Kohlstedt, H. Magnetoresistive memory with ultralow critical current for magnetization switching. Adv. Funct. Mater. 22, 4696-4703 (2012).

43 Farle, M., Mirwald-Schulz, B., Anisimov, A. N., Platow, W. \& Baberschke, K. Higher-order magnetic anisotropies and the nature of the spin-reorientation transition in face-centered-tetragonal Ni(001)/Cu(001). Phys. Rev. B 55, 3708-3715 (1997).

44 Kittel, C. Physical theory of ferromagnetic domains. Rev. Mod. Phys. 21, 541-583 (1949).

45 Vollmer, R., Gutjahr-Löser, Th., Kirschner, J., van Dijken, S. \& Poelsema, B. Spin reorientation transition in $\mathrm{Ni}$ films on $\mathrm{Cu}(001)$ : the influence of $\mathrm{H}_{2}$ adsorption. Phys. Rev. B 60, 6277-6280 (1999).

46 Stearns, M. B. in Landolt-Börnstein, New Series, Group I/I (ed. Wijn H. P. J) Vol. 19a, (Springer-Verlag, Berlin, 1986)

47 Hirth, J. P. \& Lothe, J. Theory of Dislocations, (McGraw-Hill, New York, 1968).

48 Bird, K. D. \& Schlesinger, M. Giant magnetoresistance in electrodeposited Ni/Cu and Co/Cu multilayers. J. Electrochem. Soc. 142, L65-L66 (1995).

(c) This work is licensed under a Creative Commons Attribution 4.0 International License. The images or other third party material in this article are included in the article's Creative Commons license, unless indicated otherwise in the credit line; if the material is not included under the Creative Commons license, users will need to obtain permission from the license holder to reproduce the material. To view a copy of this license, visit http:// creativecommons.org/licenses/by/4.0/ 